PREHOSPITAL CARE

\title{
Does the use of the Advanced Medical Priority Dispatch System affect cardiac arrest detection?
}

\author{
A Heward, M Damiani, C Hartley-Sharpe
}

Emerg Med J 2004;21:115-118

See end of article for authors' affiliations

......................

Correspondence to: Mr A Heward, London Ambulance Service NHS Trust, 220 Waterloo Road, London SEl 8SD, UK; andy.heward@londamb.nhs.uk

Accepted for publication 29 July 2003
Background: Cardiac arrest is the most widely recognised prehospital event that early intervention can directly affect. Chance of survival from this event decreases every minute that passes without treatment. To deliver a rapid ambulance response to these patients the early detection of cardiac arrest by control room staff is crucial. To achieve this, the London Ambulance Service (LAS) uses the Advanced Medical Priority Dispatch System. What impact has AMPDS had on identifying patients in cardiac arrest? Does compliance with AMPDS protocol influence the identification of patients in cardiac arrest?

Methods: A two stage study was undertaken. The first, compared cases coded as "cardiac arrest" and found by the responding ambulance to be in cardiac arrest before the implementation of AMPDS. This was compared with cases triaged as "cardiac arrest" and found to be in cardiac arrest across three years after AMPDS implementation. The second stage compared AMPDS compliance, over a 32 month period against the percentage of cardiac arrest calls that were found to be cardiac arrest upon the ambulance arrival. The correlation coefficient was calculated and analysed for statistical significance.

Findings: AMPDS resulted in a $200 \%$ rise in the number of patients accurately identified as suffering from cardiac arrest. A relation was identified between identification and AMPDS compliance $\left(r^{2}=0.65\right.$, $\mathrm{p}=0.001)$.

Discussion: The implementation of AMPDS increased accurate identification of patients in cardiac arrest. Additionally, the relation between factors identified suggests compliance with protocol is an important factor in the accurate recognition of patient conditions.
C ardiac arrest is probably the most widely recognised prehospital, time critical event that early interventions can directly affect. ${ }^{1}$ The chance of survival from this event reduces by between $5.5 \%^{2}$ and $10 \%^{3}$ for every minute that passes without treatment, with permanent cerebral dysfunction likely to occur three minutes after the arrest takes place. ${ }^{4}$ For these patients the likelihood of a defibrillator being used effectively decreases as the response time extends, ${ }^{5}$ with the speed of ambulance response also playing a significant factor in the chance of recovery for those patients who do not have a shockable rhythm..$^{6-8}$

Therefore, the need for a rapid ambulance response is crucial. ${ }^{5}{ }^{10}$ This factor has been identified by the Department of Health who stipulate that patients in cardiac arrest and those suffering from illness and injury that present an immediate threat to the patient's life receive an emergency response within eight minutes of the emergency call, before calls to patients suffering less serious complaints. ${ }^{11}$

To deliver this rapid response, it is important that patients suffering from a cardiac arrest are accurately identified by the ambulance service at the earliest possible opportunity. The London Ambulance Service (LAS) NHS Trust receives in excess of 90000 emergency calls per month. Because of this high volume of calls being received, there are frequently several calls waiting to be dispatched at anyone time. It is essential therefore, to highlight and prioritise patients into an order of clinical need, with patients in cardiac arrest at the top of this list. The importance now given to this area of prehospital care promotes the control centres identification of cardiac arrest as a key aspect in the first link of the "chain of survival". ${ }^{12}$ To achieve early identification, and meet the requirements of the Department of Health, the London Ambulance Service NHS Trust has been using the Advanced Medical Priority Dispatch System (AMPDS version 10) since June 1999.
AMPDS uses scripted caller interrogation protocols to provide symptom based, information to prioritise calls and allocate resources. To identify a patient as being in cardiac arrest the caller is asked, after establishing the exact location of the incident and the phone number of the caller:

- Tell me what the problem is (Tell me exactly what happened).

- Is s/he conscious

- Is $\mathrm{s} / \mathrm{he}$ breathing

A negative answer to "is s/he breathing?" or an indication that the patient is suffering agonal breathing along with a negative answer to "is s/he conscious?" indicates the patient is in cardiac arrest.

This systematic structure means that a process of continuous quality improvement (CQI), measured to an internationally set standard, can be used to ensure that the emergency medical dispatchers (EMDs) who answer the 999 emergency calls follow the scripted protocol of AMPDS and avoid asking additional, often unnecessary, questions. This process is described as compliance to AMPDS protocols, ${ }^{13}$ and, within the LAS, involves the random review of over 700 calls per month, where the emergency call is listened to by a person trained in the process of emergency call review and marked against six key areas. ${ }^{13}{ }^{14}$ The measure of compliance ensures that whether new or old, fresh faced or experienced the level of learning or education should not affect the ability to comply with the protocol.

Abbreviations: AMPDS, Advanced Medical Priority Dispatch System; $E M D$, emergency medical dispatcher; LAS, London Ambulance Service; CQI, continuous quality improvement 
In 1998 Clawson et al ${ }^{14}$ established that little consideration was given to the compliance score when evaluating AMPDS effectiveness, yet several years on, this issue still seems to be ignored. In several recent articles discussing the merits of AMPDS, such as Which? Health, and at conferences that consider the use of AMPDS for identifying calls suitable for referral to NHS Direct (and other possible agencies), compliance to the AMPDS protocols is rarely a consideration. AMPDS uses a method of CQI to ensure that AMPDS protocols are followed without digression or deviation. This provides a basis for the need for changes to be identified in conjunction with good use of the tool. ${ }^{14}$ It would be difficult to justify making a judgement about the effectiveness of a system if it is unknown whether the system is being used correctly.

There is a perception among EMDs from within London and other ambulance services that suggest that it would be better if they were left to ask their own questions and pursue routes of questioning they felt were more appropriate to the patient. This has its origins in the pre-AMPDS working practice of asking the questions that the "call taker*" felt was appropriate for that caller. This process differed between call takers and meant that no two emergency calls were asked the same set of questions. Changing to a structured process of call taking where EMDs are required to ask specific questions in a specific way was not always felt to be best practice by these staff as it did not allow them to pursue what where felt to be important lines of questioning.

To explore these issues and identify if AMPDS improved the detection of cases of patients suffering out of hospital cardiac arrest the question "Has AMPDS improved cardiac arrest detection?" was asked. Then to identify if compliance should be a consideration when discussing the use of AMPDS and to assess whether EMDs are better left to ask their own questions the question "Does compliance with AMPDS protocols affect the accuracy of cardiac arrest detection?" was posed.

\section{METHODS}

This study was undertaken in two stages. For the first step, to identify if the use of AMPDS itself has increased the detection of patients suffering from cardiac arrest, data were collected from across a four year period, before and after AMPDS implementation. Data for a one month period (April) for each of these four years were analysed. In the year before AMPDS implementation the cases coded as "cardiac arrest", "suspended" and "?Purple" (standard ambulance service terms for patients in cardiac arrest) by call takers at the time of the call were identified and compared against the cases found to be in cardiac arrest on the arrival of the responding ambulance crew.

For the year after AMPDS implementation, only those cases triaged as "cardiac arrest" at the time of the emergency call were compared against those calls found to be cardiac arrest by the responding ambulance crew. This was because calls, however defined by EMDS, are defined by the AMPDS as a subcategory "cardiac arrest" and therefore other headings were not required. The data for 1999 and 2002 were then subject to statistical analysis to identify whether a significant difference between before and after implementation existed.

For the second stage of this study, the average AMPDS compliance for each month (in excess of 700 calls per month undergo the process of CQI), over a 32 month period (June 2000 to December 2002), was entered into a Microsoft Excel spreadsheet alongside the percentage of all cardiac arrests *Those who answered emergency 999 calls to the ambulance service
pre-AMPDS implementation were referred to as call takers. After training in the use of AMPDS they have been referred to as EMDs. cases that were accurately identified by EMDs working in the communications centre. Compliance with AMPDS protocol data was a plotted against cardiac arrest identification data to create a scatter graph, with a trendline showing the relation between the two factors. The correlation coefficient was calculated using the Microsoft Excel data analysis package with the resultant significance of the correlation coefficient then being identified.

\section{FINDINGS}

Since the implementation of AMPDS there has been a rise, in excess of $200 \%$, in the accurate detection of patients suffering from cardiac arrest by EMDs working in the emergency communications centre ( $15 \%$ ( $11 \%$ to $19.5 \%$ ) v $50 \%$ (44\% to $54 \%$ ) $\mathrm{p}>0.001$ ) (fig 1 , table 1 ).

The second stage of this study identified that as compliance with AMPDS protocol increased, so the accuracy of the detection of cases of cardiac arrest cases increased (figs 1 and 2 ). Analysis identified this increase to be a positive correlation $\left(r^{2}=0.65, \mathrm{p}=0.001\right)$ showing that compliance was a significant factor in identification.

\section{DISCUSSION}

The implementation of AMPDS has had a substantial and sustained impact on the accurate identification of patients suffering from out of hospital cardiac arrest. EMDs compliance with AMPDS protocols has continued increasing the numbers of patients suffering out of hospital cardiac arrest who are accurately identified by the communications centre.

The Department of Health has set strict requirements for patients that are suffering from an illness or injury that present an immediate threat to the patients' life including cases of cardiac arrest. These categories of call require a response to the scene of an incident, within eight minutes of the emergency call being made. All other calls should have a response within 14 minutes (urban)/19 minutes (rural). Therefore, patients suffering a prehospital cardiac arrest who are triaged incorrectly may receive a significantly slower response than the eight minute intervention time beyond which a patient is unlikely to survive. ${ }^{11}$

There are additional beneficial implications in identifying patients in cardiac arrest that include increasing the chance of patient survival through the use of telephone-CPR instructions ( $t-C P R)$. Bystander CPR is frequently not undertaken at the scene of a cardiac arrest. ${ }^{15-17}$ The correct identification, though, of these cases can lead to a substantial rise in the numbers of bystanders undertaking this essential, and potentially lifesaving, intervention, through t-CPR instructions, ${ }^{16}$ which can improve survival by up to $50 \%{ }^{18}{ }^{19}$

The EMDs' faith in their individual ability to do better than the AMPDS questioning structure through their own questioning process is misguided. The risk of asking inappropriate or irrelevant questions does nothing to improve the

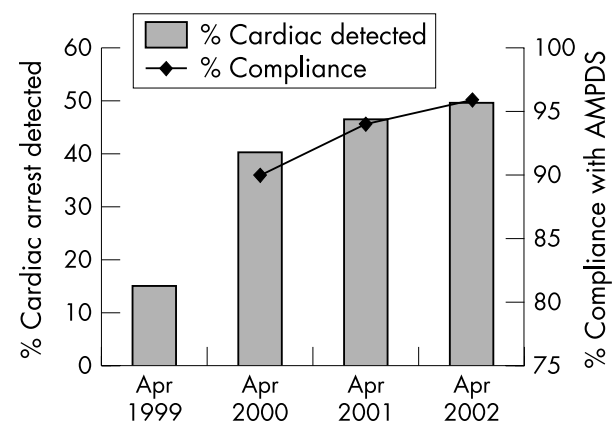

Figure 1 Increase in cardiac arrest detection through the use of AMPDS compared with compliance with AMPDS protocols for the same period. 
Table 1 Comparison of the central ambulance control diagnosis of calls found to be in cardiac arrest by the responding ambulance crew before and after AMPDS implementation

\begin{tabular}{lllll}
\hline \multicolumn{2}{l}{ Before AMPDS implementation } & & & \multicolumn{2}{l}{ After AMPDS implementation } \\
\cline { 1 - 2 } \cline { 5 - 5 } Cardiac arrest identified as & $\%$ Of calls & & Cardiac arrest identified as & $\%$ Of calls \\
\hline Collapsed state & 25 & & Cardiac arrest & 50 \\
Cardiac arrest & 15 & Unconscious & 14 \\
Difficulty in breathing & 11 & Breathing problems & 8 \\
Unconscious & 8 & Chest pain & 7 \\
Other & 6 & Unknown problems & 3 \\
Chest pain & 5 & Fitting/convulsions & 3 \\
\hline
\end{tabular}

*For the purposes of this table cardiac arrest includes the total percentage of those patients triaged as cardiac arrest, suspended, and ?purple. Comparison of data April 1999 and April 2002

identification of patients in cardiac arrest; it may even prolong the length of the call, reducing call taking capacity for unnecessary periods of time. This can also affect the time taken to provide essential dispatch life support instructions to the caller or even, more seriously, mean that the essential instructions are not given if the EMD incorrectly triages the call.

Importantly, the results show that protocol compliance should be a factor when evaluating the attributes of AMPDS in relation to its effectiveness or ability, or both, to be used for call referral to other agencies. This reinforces the assumption made by the 1994 American Position Paper ${ }^{12}$ of the importance of the EMD, and a structured protocol, in the prehospital recognition and care of patients in cardiac arrest. By making statements or basing conclusions on the findings of a system that perhaps is badly or ineffectively operated; a factor that is unknown without the level of compliance being a consideration in the findings being presented, the audience may be misguided in their interpretation of the presented information and the subsequent actions they take in relation to the effectiveness and ability of the AMPDS system.

It is highly unlikely that any system of telephone triage and/or the level of compliance with a system of call prioritisation would lead to a 100\% detection of cardiac arrest cases. Many patients deteriorate after the emergency call has been made, before the ambulance arriving on scene (unpublished data). There are also a number of reasons such as emotionally charged situations, the caller not being at the scene of an incident or the lack of understanding of the caller about the information that is required, which may lead to incomplete or inaccurate information being provided and cause the call to be inaccurately triaged. Consider also that the EMD is not immune to making mistakes and, as such, make an error and inappropriately triage the call.

In short, there are many reasons why patients in cardiac arrest may not be triaged as such by AMPDS. The one factor

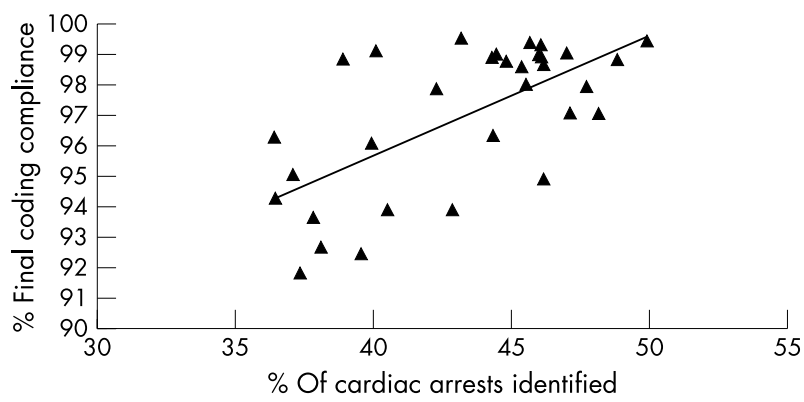

Figure 2 Correlation between AMPDS final coding compliance and cardiac arrest recognition. that does increase the rate of detection of this seriously ill group of patients is the use of a structured questioning process and the compliance of the user in following that process. In contrast with the perception of the EMD, the system is better than the person at identifying cases of cardiac arrest. The effect that compliance has on cardiac arrest detection also highlights the importance of a continuous quality improvement process to ensure that EMDs are following the protocol and that any assessment of the AMPDS is made on the basis of following the protocol occurring compared with ineffective system use. This message should be promoted and passed to ambulance services, EMDs and associated bodies to provide the patient with the greatest chance of survival and the highest level of clinical care.

\section{ACKNOWLEDGEMENTS}

Our appreciation goes to the members of the LAS' Quality Assurance Unit.

\section{CONTRIBUTORS}

Andy Heward and Chris Hartley-Sharpe developed the study concept for this paper. Michael Damiani and Andy Heward undertook the data analysis of the information and Andy Heward and Chris Hartley-Sharpe wrote this paper. Andy Heward is guarantor for this paper.

\section{Authors' affiliations}

A Heward, M Damiani, C Hartley-Sharpe, London Ambulance Service NHS Trust, London, UK

\section{REFERENCES}

1 Cummins RO, Ornato JP, Thies WH, et al. Improving survival from sudden cardiac arrest: the "chain of survival" concept. A statement for health professionals from the Advanced Cardiac Life Support Subcommittee and the Emergency Cardiac Care Committee, American Heart Association. Circulation 1991:83:1832-47.

2 Larsen MP, Eisenburg MS, Cummins RO, et al. Predicting survival from out-ofhospital cardiac arrest: a graphic model. Ann Emerg Med 1993;22:1652-8.

3 American Heart Association. 2001 Heart and stroke update. Houston, TX: American Heart Association, 2000.

4 Kumar P, Clark M. Clinical medicine, 4th edn. London: W B Saunders, 1998.

5 Pell J, Sirel JM, Marsden A, et al. Effect of reducing ambulance response times on deaths from out-of-hospital cardiac arrest: a cohort study $B M J$ 2001;322:1385-8.

6 Herlitz J, Ekstrom L, Wennerblom B, et al. Predictors of early and late survival after out-of-hospital cardiac arrest in which asystole was the first recorded arrhythmia on scene. Resuscitation 1994;28:27-36.

7 Herlitz J, Ekstrom L, Wennerblom B, et al. Survival among patients with out of hospital cardiac arrest found in electromechanical dissociation. Resuscitation 1995;29:97-106.

8 Marsden AK, Mora FM. Case report-the successful use of naloxone in an asystolic pre-hospital arrest. Resuscitation 1996;32:109-10.

9 Stueven HA, Waite EM, Troiano P, et al. Pre-hospital cardiac arrest-a critical analysis of factors affecting survival. Resuscitation 1989;17:251-9.

10 Stiell IG, Wells GA, DeMaio VJ, et al. Modifiable factors associated with improved cardiac arrest survival in a multi-centre basic life support/ 
defibrillation system: OPALS study phase I results. Ontario Pre-hospital life support. Ann Emerg Med 1999;33:44-50.

11 NHS Executive Committee. Review of ambulance performance standards, final report of steering group. London: HMSO, 1996.

12 National Institutes of Health of the United States of America. Emergency medical dispatching: rapid identification and treatment of acute myocardial infarction. Chicago: NIH Publications, no 94, 1994.

13 National Academy of Emergency Dispatch. NAED case review scoring standards for EMD-Q. 4th edn. Salt Lake City: Priority Press, 2000.

14 Clawson J, Cady G, Martin R, et al. Effect of a comprehensive quality management process on compliance with protocol in an emergency medical dispatch center. Ann Emerg Med 1998;32:578-84.
15 Gallegher EJ, Lombardi G, Gennis P. Effectiveness of bystander cardiopulmonary resuscitation and survival following out-of-hospital cardiac arrest. JAMA 1995;274:1922-5.

16 Heward A. Telephone CPR: the problems and piffalls. [Abstract]. Resuscitation 2001;55:61.

17 Hallstrum A, Cobb L, Johnson E, et al. Cardiopulmonary resuscitation by chest compression alone or with mouth-to-mouth ventilation. N Engl J Med 2000;342:1546-53.

18 Rea TD, Eisenberg MS, Culley LL, et al. Dispatcher assisted cardiopulmonary resuscitation and survival in cardiac arrest. Circulation 2001;104:2513-16.

19 Bang A, Biber B, Isaksson L, et al. Evaluation of dispatcher assisted cardiopulmonary resuscitation. Eur J Emerg Med 1999;6:175-83. 